\title{
Conocimientos de estudiantes de enfermería sobre alcohol y drogas
}

\author{
Miriam Vargas Vilela ${ }^{1}$ \\ Carla Aparecida Arena Ventura ${ }^{2}$ \\ Edilaine Cristina da Silva ${ }^{3}$
}

\begin{abstract}
Los objetivos de la presente investigación fueron verificar el conocimiento de los estudiantes de enfermería sobre el uso de alcohol y otras drogas, en especial lo relacionado a drogadicción, tolerancia, abstinencia e intoxicación; conocer las razones que los estudiantes atribuyen a la drogadicción y al inicio del uso de alcohol y otras drogas; y, el interés personal hacia el problema del consumo de drogas. Se utilizó un diseño exploratorio y descriptivo; la muestra estuvo compuesta por 44 estudiantes; se usó un cuestionario semiestructurado, construido por los investigadores con base en los objetivos propuestos, conteniendo preguntas abiertas y preguntas cerradas, totalizando 24 preguntas. Fueron seguidos los procedimientos éticos y los datos fueron sometidos a un análisis estadístico descriptivo. Se evidenció que el conocimiento de los estudiantes es todavía limitado, la comprensión, sobre las razones de los pacientes el uso y la dependencia, se mostraron incompletas, también se constató que el interés por la temática está presente.
\end{abstract}

Descriptores: Bebidas Alcohólicas; Drogas Ilícitas; Estudiantes de Enfermería.

\footnotetext{
${ }_{1}$ Profesor de la Carrera de Enfermería, Universidad Mayor de San Andrés, La Paz, Bolivia. E-mail: miriam_vilela2004@yahoo.com.

2 Profesor Doctor, Escola de Enfermagem de Ribeirão Preto, Universidade de São Paulo, Centro Colaborador de la OMS para el Desarrollo de la Investigación en Enfermería, Brasil. E-mail: caaventu@eerp.usp.br.

${ }_{3}^{3}$ Profesor Doctor, Escola de Enfermagem de Ribeirão Preto, Universidade de São Paulo, Centro Colaborador de la OMS para el Desarrollo de la Investigación en Enfermería, Brasil. E-mail: nane@eerp.usp.br.
}

Correspondencia:

Edilaine Cristina da Silva

Universidade de São Paulo. Escola de Enfermagem de Ribeirão Preto

Av. Bandeirantes, 3900

Bairro Monte Alegre

CEP: 14040-902 Ribeirão Preto, SP, Brasil

E-mail: nane@eerp.usp.br 


\section{Conhecimentos de estudantes de enfermagem sobre álcool e drogas}

O objetivo da presente pesquisa foi verificar o conhecimento de estudantes de enfermagem sobre o uso de álcool e outras drogas, em especial a drogodependência, tolerância, abstinência e intoxicação; as razões que os estudantes atribuem à drogodependência e ao início do uso de álcool e outras drogas e o interesse pessoal na problemática do consumo de drogas. Utilizou-se um desenho descritivo-exploratório; amostra composta por 44 estudantes; um questionário semiestruturado, construído pelos pesquisadores com base nos objetivos propostos, contendo perguntas abertas e fechadas, totalizando 24 questões. Foram seguidos os procedimentos éticos e os dados foram submetidos à análise descritiva exploratória. Evidenciou-se que o conhecimento dos estudantes é ainda, limitado, a compreensão sobre as razões dos pacientes para o uso e a dependência é incompleta e o interesse pelo assunto mostra-se evidente.

Descritores: Bebidas Alcoólicas; Drogas Ilícitas; Estudantes de Enfermagem.

\section{Nursing Students' Knowledge about Alcohol and Drugs}

The aim of this study was to verify the knowledge of nursing students regarding the use of alcohol and other drugs, particularly addiction, tolerance, withdrawal and intoxication, the reasons students give for drug addiction and commencement, and personal interest in the issue of drug use. A descriptive-exploratory design was used, with a sample of 44 students, by applying a semi-structured questionnaire, constructed by the researchers based on the objectives, with open and closed questions, totaling 24 points. Ethical procedures were followed and data were submitted to exploratory descriptive analysis. It was shown that student's knowledge is still limited, comprehension about a patient's reasons for using and becoming addicted is incomplete and the interest is current.

Descriptors: Alcoholics Beverages; Street Drugs; Students, Nursing.

\section{Introducción}

El uso de substancias psicoactivas es un problema generalizado que afecta a toda la población del mundo, sea a través de las enfermedades relacionadas al uso del alcohol, tabaco y otras drogas, sea por las consecuencias sociales del abuso o del tráfico de drogas ilícitas ${ }^{(1)}$, afectando a toda la sociedad, especialmente a los jóvenes. Los crecientes índices de consumo de drogas, en especial en la población infanto-juvenil, y las respuestas insuficientes frente a este problema requieren nuevas acciones coordinadas de los diferentes estratos de la sociedad.

En esta dinámica, tanto las universidades como las organizaciones públicas y privadas del área de la salud, en particular las facultades, carreras y escuelas de Enfermería, en Bolivia, desarrollan trabajos orientados hacia la promoción, prevención y reinserción social en el tema de alcohol y drogas ${ }^{(2-4)}$.
El consumo de substancias psicoactivas sigue una tendencia progresiva en relación a la magnitud y a los daños causados a la sociedad debido al uso indiscriminado. En ese contexto, el profesional enfermero es considerado un agente clave en programas de promoción, prevención y reducción en el enfrentamiento del fenómeno de las drogas $^{(5)}$.

Una investigación realizada con estudiantes de enfermería apuntó que estos encuentran dificultades para planificar estrategias de intervención en el contexto de uso indebido de alcohol y drogas. Ante una propuesta de promoción de la salud, la tendencia biologisista de la universidad, propia del modelo médico, constituye un obstáculo para la articulación entre la teoría e la práctica de estos estudiantes ${ }^{(6)}$.

Sin embargo, los contenidos sobre alcohol y drogas se encuentran dispersos en diferentes asignaturas de la 
carrera de enfermería de la Universidad Mayor de San Andrés. Con el objetivo de alterar esta situación, en los últimos cinco años, se está realizado un proyecto de inserción de contenidos referentes al uso y abuso de drogas, con apoyo de la Comisión Interamericana para el Control del Abuso de Drogas de la Organización de los Estados Americanos (OEA-CICAD).

Ante los cambios, surgió la necesidad de revelar datos que permitirán una retroalimentación para la institución, traducida en una propuesta para el mejoramiento del plan de estudios de Enfermería, enfatizando el desarrollo de competencias, habilidades sociales, capacidades técnicas y sociales para enfrentar situaciones de salud que involucran el alcohol y otras drogas.

Así, el objetivo de la presente investigación fue verificar: 1) el conocimiento de los estudiantes de enfermería sobre el uso de alcohol y otras drogas, en especial la drogadicción, tolerancia, abstinencia y intoxicación; 2) las razones que los estudiantes atribuyen a la drogadicción y al inicio del uso de alcohol y otras drogas; 3 ) el interés personal hacia el problema del consumo de drogas.

\section{Material y métodos}

Consiste en un estudio exploratorio y descriptivo, caracterizado por examinar un tema de investigación poco estudiado o poco abordado anteriormente ${ }^{(7)}$.

La muestra fue compuesta por todos (100\%) los estudiantes, totalizando 44, los cuales participaban del internado rotatorio de pregrado en Enfermería de la Universidad Mayor de San Andrés, involucrados en actividades de docencia. Como criterios de inclusión se consideró el interés y disponibilidad de participación en el estudio.

El instrumento utilizado para recolección de datos fue un cuestionario semiestructurado, construido por los investigadores con base en los objetivos propuestos, conteniendo preguntas abiertas y cerradas, totalizando 24 preguntas. Las preguntas enfatizaron el conocimiento de los estudiantes de enfermería sobre el uso de alcohol y otras drogas, en especial la drogadicción, tolerancia, abstinencia y intoxicación; las razones que ellos atribuyen a la drogadicción y al inicio del uso de alcohol y otras drogas; y el interés personal hacia el problema del consumo de drogas.
En la construcción del cuestionario se consideró que ese instrumento constituía una forma concreta de la técnica de observación, logrando que el investigador fije su atención en ciertos aspectos y se sujete determinadas condiciones. Así, el cuestionario contiene los aspectos del fenómeno que se consideran esenciales, reduciendo la realidad a cierto número de datos esenciales y precisando el objeto de estudio.

Con el propósito de validar el instrumento de recolección de datos fue realizada la validación respecto a su utilidad técnica para darle el rigor científico.

La compilación de los datos ocurrió entre septiembre de 2006 y enero de 2007

En la etapa de aplicación del cuestionario se siguieron todos los procedimientos éticos pertinentes y todos los requisitos exigidos por la Escuela de Enfermería. Los participantes fueron informados sobre todos los aspectos de la investigación y manifestaron su consentimiento mediante la firma del Término de Conocimiento Libre y Aclarado (TCLA). El proyecto también fue presentado al Honorable Consejo de Carrera para su respectiva consideración y aprobación.

Para la recolección de datos fueron invitados los estudiantes del internado rotatorio, que se encontraban en diferentes etapas de práctica clínica y en el servicio social rural obligatorio. Fue realizada una reunión con el grupo a fin de informar sobre la naturaleza y objetivo de la investigación y mediante el acuerdo en participar se procedió a la entrega del cuestionario semiestructurado para su respectivo llenado.

Los datos fueron analizados con un paquete estadístico. Se procedió al estudio descriptivo observando la distribución de los datos respecto a su naturaleza. En especial, las cuestiones sobre el conocimiento fueron analizadas basándose en las definiciones actuales de drogadicción, tolerancia, abstinencia e intoxicación para atribución de los conceptos correcto e incorrecto a las respuestas.

\section{Resultados}

El cuestionamiento sobre los conceptos relacionados al uso de alcohol y otras drogas resultó en $88,9 \%$ de respuestas correctas de los estudiantes sobre drogadicción, en $61,1 \%$ sobre la tolerancia, en 97,2 sobre la abstinencia, y en $89.0 \%$ sobre la intoxicación (Tabla 1). 
Tabla 1 - Distribución de las respuestas de los estudiantes sobre conceptos relacionados al uso de alcohol y drogas, La Paz, Bolivia 2007

\begin{tabular}{lcc}
\hline \multicolumn{1}{c}{ Concepto(n=36) } & Frecuencia Total & $\%$ \\
\hline Drogadicción & 32 & 88,9 \\
Correcto & 4 & 11,1 \\
Incorrecto & 0 & 0,0 \\
No contestaron & & \\
Tolerancia & 22 & 61,1 \\
Correcto & 6 & 16,7 \\
Incorrecto & 8 & 22,2 \\
No contestaron & & \\
Abstinencia & 35 & 97,2 \\
Correcto & 0 & 0,0 \\
Incorrecto & 1 & 2,8 \\
No contestaron & & \\
Intoxicación & 32 & 89,0 \\
Correcto & 2 & 5,5 \\
Incorrecto & 2 & 5,5 \\
No contestaron & 36 & 100 \\
Total & & \\
\hline
\end{tabular}

En la opinión de los estudiantes, el inicio del uso de alcohol y drogas ocurre principalmente por influencia de grupos y por curiosidad, ambas respuestas con un 36,1\%, como demuestra la Tabla 2 .

Tabla 2 - Distribución de las repuestas de los estudiantes sobre las razones para el inicio del uso y la drogadicción, La Paz, Bolivia 2007

\begin{tabular}{lcc}
\hline \multicolumn{1}{c}{ Variable (n=36) } & $\begin{array}{c}\text { Frecuencia } \\
\text { Total }\end{array}$ & $\%$ \\
\hline Inicio & 13 & 36,1 \\
Por influencia de grupos & 13 & 36,1 \\
Por curiosidad & 1 & 2,8 \\
Por influencia de familiares & 1 & 2,8 \\
Por timidez & 6 & 16,7 \\
Por sufrir algún problema emocional & 2 & 5,6 \\
No contestaron & & \\
Drogadicción & 9 & 25,0 \\
Falta de carácter & 9 & 25,0 \\
Personalidad débil & 8 & 22,2 \\
Hábito cultural & 1 & 2,8 \\
Origen genética & 6 & 16,7 \\
Otros & 3 & 8,3 \\
No contestaron & 36 & 100 \\
Total & &
\end{tabular}

Conforme se presenta en la Tabla 1, a respecto de la pregunta "la adicción al alcohol y drogas es provocada por", los estudiantes apuntaron predominantemente la falta de carácter (25\%), personalidad débil (25\%) y hábito cultural (22.2\%) como las principales razones para la adicción al alcohol y otras drogas.

Sobre la identificación de características de dependencia, 25 (69.4\%) estudiantes relataron poder identificar las características de dependencia del alcohol y $25 \%$ consideran poder identificar las características de dependencia de otras drogas.

La experiencia de tratar personas con problemas de dependencia de alcohol fue apuntada por 23 (55.6\%) estudiantes, mientras solamente 10 (27.8\%) trataron personas con problemas de dependencia de otras drogas.

Entre los estudiantes entrevistados, 30 (83.3\%) respondieron que la adquisición de conocimientos sobre las drogas y alcohol fue de utilidad en el ámbito personal y $33(91,7 \%)$ indicaron que los contenidos de alcohol y drogas deben ser mantenidos en el plan de estudios de enfermería.

La mejor instancia para adquisición de conocimientos sobre alcohol y otras drogas en la opinión de los estudiantes fue el abordaje teórico durante la formación de enfermería $(50,0 \%)$, los medios de información $(19,4 \%)$, el abordaje práctico $(13,9 \%)$ y la comunicación con los padres $(8,3 \%)$. En este sentido, el primer año de estudio fue apuntado por los estudiantes como el mejor año para adquirir y asimilar conocimientos sobre la temática de alcohol y drogas.

Sobre las estrategias de atención de enfermería, los estudiantes indicaron la orientación al paciente $(38,9 \%)$, la orientación a la familia $(11,1 \%)$, las terapias de grupo $(19,4 \%)$, los cuidados básicos de enfermería $(8,3 \%)$ y otros el $11,1 \%$.

Las estrategias de enfermería en la comunidad más apuntadas por los estudiantes incluyeron identificación de grupos vulnerables $(72,2 \%)$ y incentivo a actividades comunitarias deportivas y culturales, sin uso de substancias $(16,7 \%)$.

El cuestionamiento sobre el interés personal hacia el problema del consumo de drogas resultó afirmativa por $31(86,1 \%)$ entrevistados. $80,6 \%$ (29) de los estudiantes contestaron que el enfermero en el equipo multidisciplinario puede promover una mejora en la calidad de vida de usuarios de alcohol y drogas.

\section{Discusión}

Con respecto al inicio del consumo de alcohol y drogas, los estudiantes identifican la influencia del grupo y la curiosidad como principales factores que influyen en el inicio, seguido por la presencia de problemas de 
naturaleza emocional. Estos problemas también son considerados factores de riesgo en la literatura sobre el tema(8-10); los mismos están ligados a la dimensión individual de las personas y se refieren al bajo amor propio, pobre autocontrol, manejo de las aptitudes sociales inadecuado, sensación de búsqueda, depresión, ansiedad y eventos estresantes en la vida.

Las respuestas sobre las razones para la adicción indicaron que entre los estudiantes predominan percepciones negativas sobre el dependiente. Asimismo, se observó la percepción del estudiante sobre el factor cultural del consumo de drogas al indicar el hábito cultural como justificativa para la adicción. Estos datos indican la necesidad de trabajar los valores y actitudes de los estudiantes a fin de obtener la adecuada formación profesional.

Los resultados sobre los conceptos relacionados al uso de alcohol y drogas indicaron que la mayoría de los estudiantes son capaces de elaborar un concepto teórico sobre drogadicción, abstinencia e intoxicación y que tuvieron menos respuestas correctas sobre el concepto de tolerancia. Sin embargo, hay un porcentaje de estudiantes que presentó respuestas incorrectas, indicando que el aprendizaje no alcanzó la totalidad de los estudiantes. Hay en este punto una indicación de que el conocimiento necesita avanzar de la esfera cognitiva para la acción. En este sentido, una investigación desarrollada con estudiantes de enfermería, reveló que el conocimiento se va construyendo progresivamente y generalmente empieza por la etapa cognitiva(11).

Sin embargo, en un estudio anterior, los estudiantes presentaron escasos conocimientos referentes a la drogadicción. Sus conceptos eran superficiales e impregnados de conocimiento intuitivo. Los autores creen que el abordaje de estos contenidos puede haber sido superficial o que los estudiantes no otorgaron la debida importancia a la temática ${ }^{(6)}$. En este caso, la respuesta contundente de los estudiantes respecto a que los contenidos sobre alcohol y drogas deben ser mantenidos en el plan de estudios de la carrera de enfermería refleja que ellos consideran la necesidad de la temática para lograr un mejor desempeño profesional.

El bajo número de estudiantes que apuntaron saber identificar características específicas que demuestren dependencia de las drogas y alcohol denota la necesidad de incrementar las oportunidades para el aprendizaje de esta competencia, incluyéndose contacto con estos pacientes y discusiones de casos clínicos orientados. En este sentido, los casos clínicos han sido utilizados con éxito en la enseñanza de contenidos del área de salud mental, pues estimulan la participación del estudiante, además de permitir el acceso a diferentes niveles de competencia(11).

La valorización del abordaje teórico en detrimento del abordaje práctico puede estar relacionada a una dificultad del estudiante ${ }^{(12)}$ en problematizar su práctica y buscar el aprendizaje de una manera más autónoma e independiente.

El reconocimiento de que el enfermero es un profesional importante en el equipo multidisciplinario para promover una mejoría en la calidad de vida de usuarios de alcohol y drogas, contribuye para la determinación del rol del enfermero en el concepto de estos estudiantes, que pueden reconocer más fácilmente la responsabilidad del profesional enfermero con los problemas del uso de alcohol y otras drogas.

\section{Conclusiones}

El estudio presentó como limitación la falta de datos sociodemográficos en el cuestionario.

Las conclusiones más relevantes del presente estudio conducen a enfatizar que los procesos de capacitación que tuvieron los estudiantes del internado rotatorio de pregrado en Enfermería de la Universidad Mayor de San Andrés, sobre la temática de drogas y alcohol, fueron un factor importante para la adquisición de conocimientos. Sin embargo, el estudio evidenció que el conocimiento es todavía limitado, la comprensión sobre las razones de los pacientes hacia el uso y la dependencia es incompleta y el interés por la temática está presente. Delante de los resultados, se sugiere realizar inversiones en otras investigaciones que tengan por objetivo analizar el proceso educativo en la enseñanza del uso y abuso de drogas, con la finalidad de encontrar mecanismos pedagógicos que garanticen el aprendizaje efectivo.

Finalmente, se puede concluir que este trabajo contribuye con informaciones necesarias a la formación de los profesionales de Enfermería para enfrentar de manera acertada y con competencias técnicas adecuadas las demandas de la sociedad frente al problema de alcohol y drogas, considerando las especificidades de Bolivia, como un país multilingüe, pluricultural y multiétnico.

\section{Agradecimientos}

Agradecemos a la Comisión Interamericana para el Control del Abuso de Drogas/CICAD de la Secretaria de Seguridad Multidimensional/SSM de la Organización de 
Ios Estados Americanos/OEA, la Secretaria Nacional de Políticas sobre Drogas/SENAD do Gabinete de Seguridad Institucional/Brasil, la Escuela de Enfermería de Ribeirao Preto de la Universidad de Sao Paulo y Centro Colaborador de la Organización Mundial de la Salud para el Desarrollo de la Investigación en Enfermería, la población representada en los estudios de investigación, bien como a las autoridades de las universidades representadas por los participantes del Programa En-Line de Especialización en Investigación sobre el Fenómeno de las Drogas - PREINVEST, periodos 2005, 2006, 2007 y 2008.

\section{Referencias}

1. Desjarlais R, Eisenberg L, Good B, Kleinman A. Salud Mental en el Mundo: Problemas y Prioridades en Poblaciones de Bajos Ingresos. Washington (DC): Organización Panamericana de la Salud; 1997.

2. Castillo FA del. El uso indebido de drogas en estudiantes de Bolivia 1993-1996-1999. Psicoativa 2000; 18:53-70.

3. Flores IEE, Luis MAV. Uso y actitudes relacionado a las drogas en las estudiantes de enfermería de la Universidad Mayor de San Andrés. Rev. Latino-Am. Enfermagem. 2004 março-abril; 12(número especial):376-82.

4. Oliveira MR, Luis MAV. Factores de riesgo para el consumo de alcohol en escolares de 10 a 18 años, de establecimientos educativos fiscales en la ciudad de La Paz - Bolivia (2003 2004). Rev. Latino-Am. Enfermagem. 2005 setembro-outubro; 13(número especial):880-7.

5. Gelbcke F, Souza Padilla MI. O fenômeno das drogas no contexto da promoção da saúde. Texto \& contexto enferm 2002 abril/junho 2002; 13(2):273-8.

6. Rojo MD, Bueno SMV, Silva EC. Concepção dos estudantes de enfermagem sobre promoção da saúde relacionada ao uso de substâncias psicoativas. Rev. Latino-Am. Enfermagem. 2008 maio-junho; 16(especial).
7. Dankhe GL. Investigación y comunicación. In: Dankhe GL, organizador. La comunicación humana ciencia social. México (DC): Mc.Graw-Hill, 1976, p. 385-454.

8. Ruiz MR, Andrade D. Family and risk factors related to alcohol consumption and smoking among children and adolescents. Rev. Latino-Am. Enfermagem. 2005; (13):813-8.

9. Bandura A. Self-efficacy: The exercise of control. New York (NY): W.H. Freeman; 1977.

10. Becoña E. Bases científicas de la prevención de las drogodependencias. Madri: Universidad de Santiago de Compostela Delegación del Gobierno para el Plan Nacional sobre Drogas, 2002, p.15-7.

11. Silva EC, Furegato ARF, Godoy S. Clinical case studies in mental health through on-line discussion. Rev. Latino-Am. Enfermagem. 2008 maio-junho; 16(3):425-31.

12. Batista BA. Formación del académico en oficio de enfermería y su contacto con las drogas psicoactivas. Texto \& contexto enferm, 2004; 13(2):241-54. 